\title{
Effects of pressing cycles and durations on the properties of compreg oil palm wood
}

\begin{abstract}
Oil palm wood (OPW) extracted from the oil palm trunks and subjected to a four-step compreg method consisted of drying, impregnation, re-drying and hot-pressing densification process. For the densification process, four different pressing cycles (involving 1 ï 3 cycles) were proposed and conducted with three pressing durations (30,35 and $40 \mathrm{~min}$ ). Properties of the compreg OPW were evaluated. The results revealed that the samples compressed using the pressing process with more than 1 cycle displayed significantly better water absorption and thickness swelling. OPW samples that compressed with longer pressing durations also reported better performance and the formaldehyde emission decreased along with extending pressing durations. However, the improvement was not statistically significant, therefore, 30 min pressing duration is sufficient to achieve satisfied properties.
\end{abstract}

Keyword: Oil palm wood; Pressing cycles; Pressing durations; Compreg; Impregnation 